\title{
Covalent Immobilization of 'Caged' Liquid Crystal Microdroplets on Surfaces
}

\author{
Xuanrong Guo, ${ }^{1, \dagger}$ Uttam Manna, ${ }^{1, f, \dagger}$ Nicholas L. Abbott, ${ }^{1, *}$ and David M. Lynn ${ }^{1,2, *}$
}

${ }^{l}$ Department of Chemical and Biological Engineering, 1415 Engineering Drive, and ${ }^{2}$ Department of Chemistry, 1101 University Avenue, University of Wisconsin - Madison, Madison, Wisconsin 53706. ${ }^{\# C u r r e n t ~ a d d r e s s: ~ D e p a r t m e n t ~ o f ~ C h e m i s t r y, ~ I n d i a n ~ I n s t i t u t e ~ o f ~}$ Technology-Guwahati, Guwahati, Assam-781039, India. 'These authors contributed equally.

\section{Supporting Information}

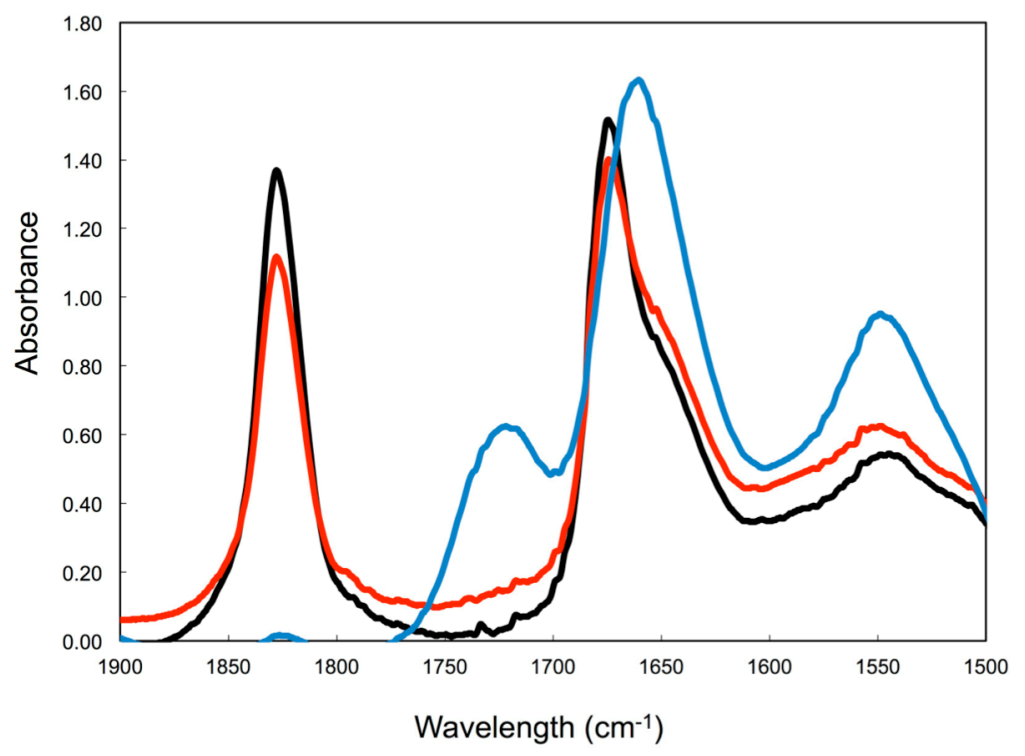

Figure S1: Polarization-modulation infrared reflectance-absorbance spectra (PM-IRRAS) of PEI/PVDMA multilayers terminated with a final layer of PEI (see text) fabricated on planar, gold-coated silicon wafers. Shown are spectra of a film before (black line) and after (red and blue lines) incubation at room temperature in deionized water for one hour (red) or 24 hours (blue). Inspection of the red curve reveals only a small decrease in the carbonyl peak at 1828 $\mathrm{cm}^{-1}$ (corresponding to the carbonyl stretch of the azlactone group in PVDMA) after one hour, indicating that the azlactone groups in the film did not hydrolyze to a significant extent under these conditions. Inspection of the blue curve reveals the nearly complete disappearance of the characteristic peak at $1828 \mathrm{~cm}^{-1}$ and the appearance of a peak at $1729 \mathrm{~cm}^{-1}$ (corresponding to the carbonyl stretch of the carboxylic acid group of a hydrolyzed azlactone group) after 24 hours, indicating that the azlactone functionality in the films was almost completely hydrolyzed under these conditions. 


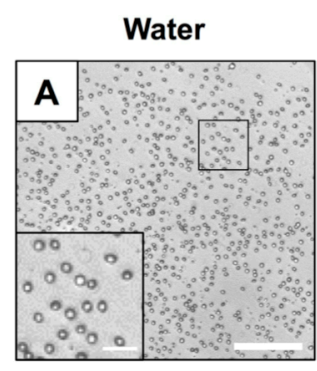

$150 \mathrm{mM} \mathrm{NaCl}$
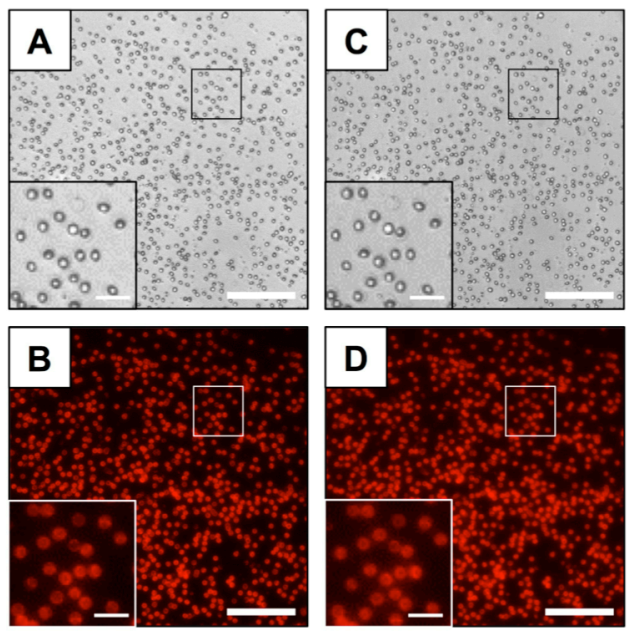

$1 \mathrm{M} \mathrm{NaCl}$

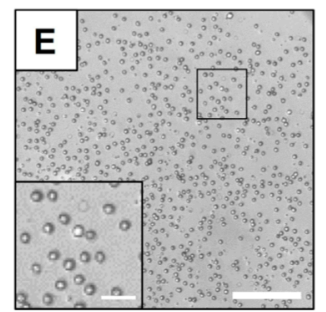

$5 \mathrm{M} \mathrm{NaCl}$
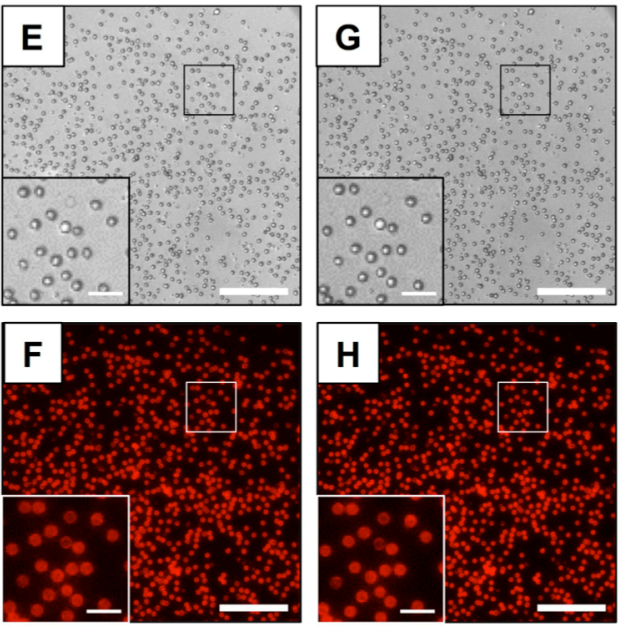

15 mM Triton-X100
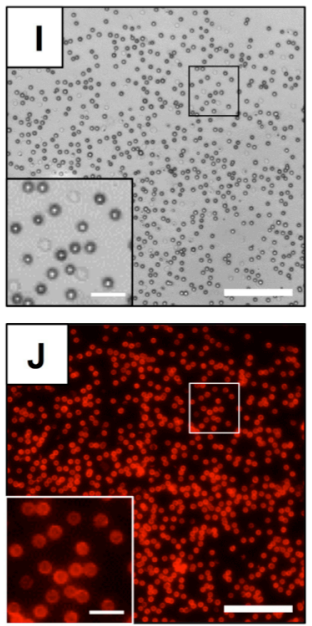

Figure S2: This figure is a companion to Figure 4 of the main text, and shows results after rinsing 'caged' LCs immobilized on amine-reactive surfaces using additional conditions (see main text). (A-J) Bright-field (top row) and fluorescence (bottom row) microscopy images of an amine-reactive surface after treatment with dispersions of PEI-functionalized capsules for one hour and washing with conditions described below. All micrographs within each group were acquired in the same field of view. Panels show the surface after rinsing with (A-B) deionized water, (C-D) $150 \mathrm{mM} \mathrm{NaCl}$, (E-F) $1.0 \mathrm{M} \mathrm{NaCl}$, (G-H) $5.0 \mathrm{M} \mathrm{NaCl}$, and (I-J) 15 $\mathrm{mM}$ TritonX-100. Scale bars are $120 \mu \mathrm{m}$ ( $20 \mu \mathrm{m}$ for insets). 

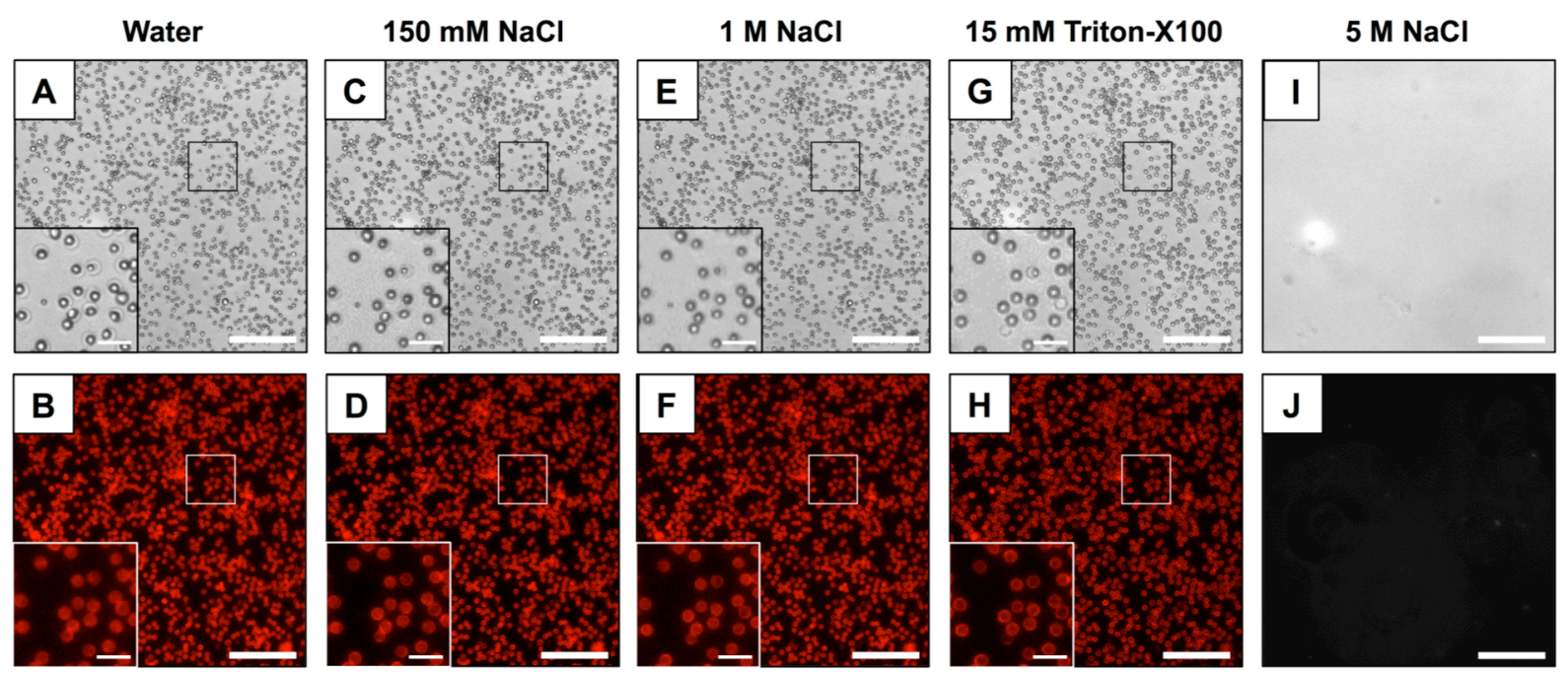

Figure S3: This figure is a companion to Figure 4 of the main text, and shows results after rinsing 'caged' LCs immobilized on anionic surfaces using additional conditions (see main text). (A-J) Bright-field (top row) and fluorescence (bottom row) microscopy images of an anionic surface after treatment with dispersions of PEI-functionalized capsules for one hour and washing with conditions described below. All micrographs within each group were acquired in the same field of view. Panels show the surface after rinsing with (A-B) deionized water, (C-D) $150 \mathrm{mM} \mathrm{NaCl}$, (E-F) $1.0 \mathrm{M} \mathrm{NaCl}$, (G-H) $15 \mathrm{mM}$ TritonX-100, and (I-J) $5.0 \mathrm{M}$ $\mathrm{NaCl}$. Scale bars are $120 \mu \mathrm{m}(20 \mu \mathrm{m}$ for insets). 'Caged' LCs remained immobilized on anionic surfaces after gentle rinsing with $1.0 \mathrm{M} \mathrm{NaCl}$, as shown in panels (E-F), but be could partially or completely dislodged with more vigorous rinsing with $1.0 \mathrm{M} \mathrm{NaCl}$ (not shown). 'Caged' LCs were completely dislodged from anionic surfaces after gentle rinsing with $5.0 \mathrm{M}$ $\mathrm{NaCl}$ (I-J), but remained substantially in place even after vigorous rinsing with $150 \mathrm{mM} \mathrm{NaCl}$ (C-D). 

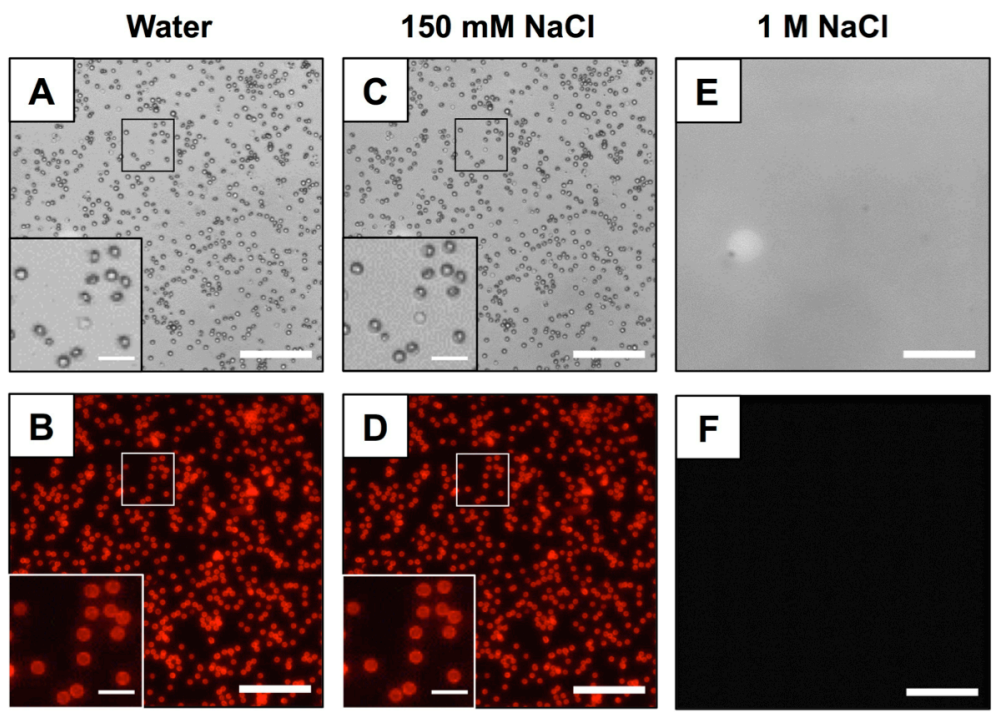

Figure S4: Bright-field (top row) and fluorescence (bottom row) microscopy images of a bare glass surface after treatment with a dispersion of PEI-functionalized capsules for one hour and washing with conditions described below. All micrographs within each group were acquired in the same field of view. Panels show the surface after rinsing with (A-B) deionized water, (C-D) $150 \mathrm{mM} \mathrm{NaCl}$, and (E-F) $1.0 \mathrm{M} \mathrm{NaCl}$. Scale bars are $120 \mu \mathrm{m}$ (20 $\mu \mathrm{m}$ for insets). 

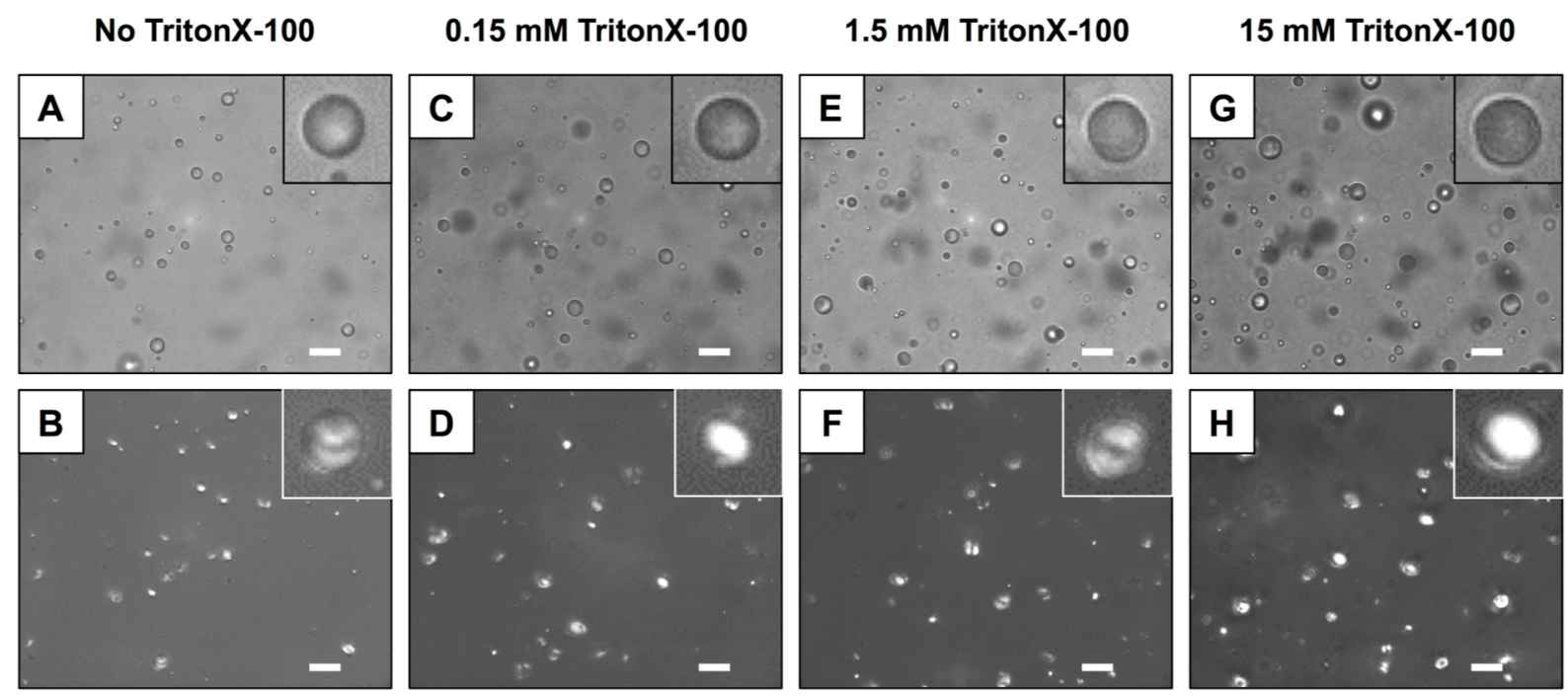

Figure S5: Bright-field (top row) and polarized light (bottom row) microscopy images of bare E7 LC-in-water emulsion droplets dispersed in water (A-B) before and $(\mathrm{C}-\mathrm{H})$ after the addition of TritonX-100 at concentrations of (C-D) $0.15 \mathrm{mM}$, (E-F) $1.5 \mathrm{mM}$, and (G-H) 15 $\mathrm{mM}$. These droplets of E7 are identified to be in the bipolar configuration, as indicated by the presence of two point defects at opposite poles of the droplets and their corresponding optical appearances under polarized light; these conditions and optical appearances differ substantially from those of LC droplets present in the radial configuration (see text). The critical micelle concentration (CMC) of TritonX-100 is 0.22 to $0.24 \mathrm{mM}$. Scale bars are 10 $\mu \mathrm{m}$. For polarized light microscopy images, the direction of the crossed polarizers was parallel and perpendicular to the scale bars shown. 

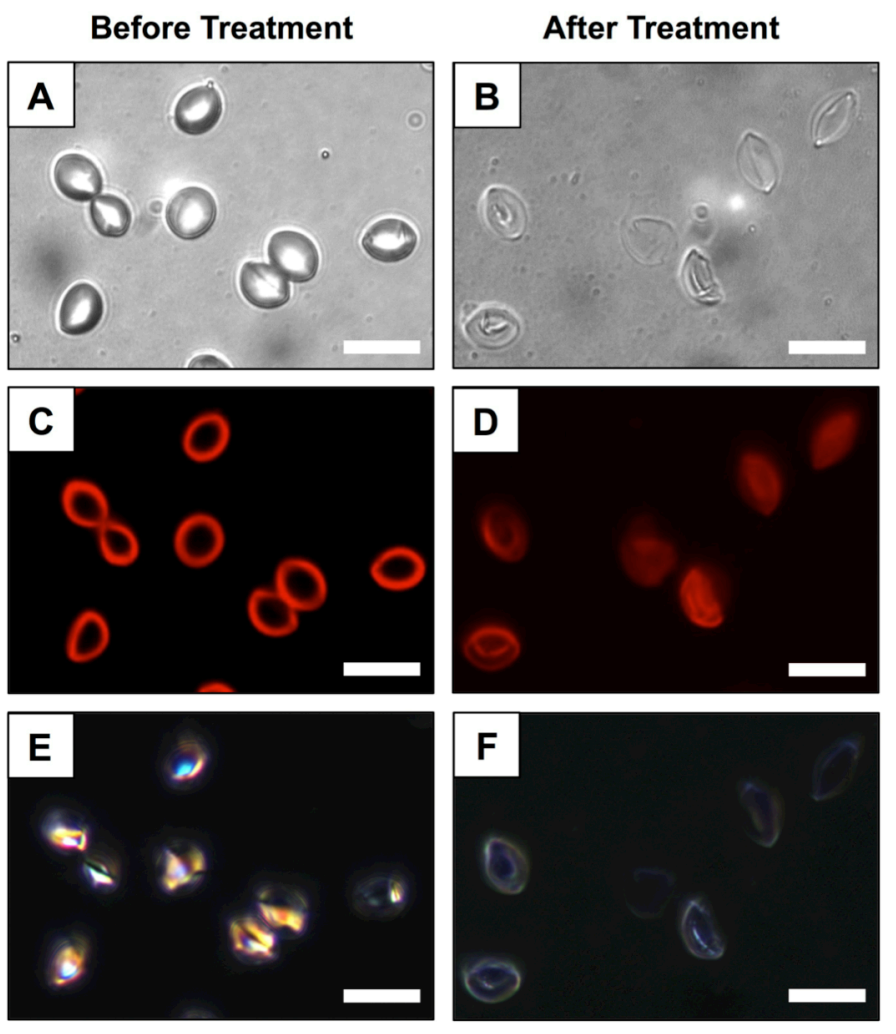

Figure S6: Bright-field (top row), fluorescence (middle row), and polarized light (bottom row) microscopy images of immobilized 'caged' LC droplets (A,C,E) after drying and then (B,D,F) after incubation in an oven at $\sim 70{ }^{\circ} \mathrm{C}$ for two hours (the images in $(\mathrm{B}, \mathrm{D}, \mathrm{F})$ were collected after the sample was cooled back down to room temperature). Inspection of panels $(\mathrm{B}, \mathrm{D}, \mathrm{F})$ reveals collapsed capsules with negligible birefringence, indicating that the LC 'caged' in the immobilized capsules leaked out after heating the LC above its clearing temperature $\left(\sim 60{ }^{\circ} \mathrm{C}\right)$. Scale bars are $10 \mu \mathrm{m}$. For polarized light microscopy images, the direction of the crossed polarizers was parallel and perpendicular to the scale bars shown. 


\section{Supporting Videos:}

Video S1: Video acquired using bright-field and polarized light microscopy showing changes in the mobilities of three LC droplets in 'caged' LC's immobilized covalently on a planar surface. The droplets in their initial states wet the inner surfaces of the surrounding polymer capsules and are immobile. The addition of DTAB $(1.0 \mathrm{mM})$ to the surrounding medium results in a change in droplet shape to more spherical as well as a change in droplet mobility that results in droplet rotation and translation within the confines of the surrounding polymer cages. Changes in mobility upon the addition of surfactant occurs simultaneously with a transition from the bipolar to radial configuration. The video shown here has been adjusted to run at $30 \mathrm{X}$ the original speed and shows video acquired in the following sequence: (i) bright-field before addition of DTAB, (ii) polarized light before addition of DTAB, (iii) polarized light after the addition of DTAB, and (iv) bright-field after the addition of DTAB. See main text for additional details of these experiments. 